\title{
Aplicativos móveis para as sociedades menos favorecidas
}

Mobile apps for disadvantaged societies

Aplicaciones móviles para las sociedades menos favorecidas

Janize Silva Maia1 ${ }^{10}$ https://orcid.org/0000-0001-5939-3353 Heimar de Fátima Marin² iD https://orcid.org/0000-0003-3670-7502

Como citar:

Maia JS, Marin HF. Aplicativos móveis para as sociedades menos favorecidas. Acta Paul Enferm. 2021;34:eAPE002214.

DOI

http://dx.doi.org/10.37689/actaape/2021AR02214

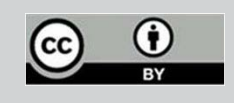

Descritores

Aplicativos móveis; Tecnologia biomédica; Acesso

à informação

Keywords

Mobile applications; Biomedical technology; Access

to information

Descriptores

Aplicaciones móviles; Tecnología biomédica; Acceso a la información

Submetido

31 de Julho de 2019

Aceito

18 de Dezembro de 2020

Autor correspondente

Janize Silva Maia

E-mail: janizecs@yahoo.com.br

\section{Resumo}

Objetivo: Descrever os impactos dos aplicativos móveis diretamente em favor da saúde das comunidades menos favorecidas que vivem em países de baixa renda ou em desenvolvimento e, indiretamente, por meio dos profissionais de saúde que prestam assistência nestas áreas.

Métodos: Revisão integrativa da literatura nas bases de dados PubMed, SciELO e LILACS de pesquisas originais baseadas em evidências, publicadas entre 2010 e 2019 disponíveis na íntegra e em português e inglês, mediante a questão norteadora: "Qual o impacto dos aplicativos móveis no cuidado direto e indireto em saúde em populações de baixa renda e em desenvolvimento?", a partir dos descritores: tecnologia biomédica, informação em saúde e aplicativos móveis, sendo este último presente em todos os cruzamentos. A estratégia de busca, seleção e categorização dos estudos foi realizada pela leitura de um pesquisador.

Resultados: Os aplicativos demonstraram benefícios quanto ao diagnóstico de doenças, adesão dos usuários ao tratamento, acompanhamento dos pacientes pelos profissionais de saúde e autocuidado, constituindo uma importante ferramenta para o monitoramento e gerenciamento de doenças.

Conclusão: A tecnologia móvel tem o potencial de melhorar os serviços de atenção primária por meio da qualificação dos cuidados em saúde promovidos pelos profissionais e acesso direto do usuário, sobretudo em países de baixa renda, cujos indicadores de saúde são preocupantes.

\section{Abstract}

Objective: To describe the impacts of mobile applications directly in favor of the health of disadvantaged communities living in low-income or developing countries and, indirectly, through healthcare professionals providing assistance in these areas.

Methods: This is an integrative literature review in the PubMed, SciELO and LILACS databases of original evidence-based research, published between 2010 and 2019, available in full and in Brazilian Portuguese and English, through the guiding question: "What is the impact of mobile applications on direct and indirect health care in low-income and developing populations?". The following descriptors were used: biomedical technology, health information and mobile applications, the latter being present in all intersections. The strategy of search, selection and categorization of studies was performed by the reading of a researcher.

Results: The applications demonstrated benefits regarding the diagnosis of diseases, users' treatment adherence, patient follow-up by health professionals and self-care, constituting an important tool for monitoring and managing diseases.

Conclusion: Mobile technology has the potential to improve primary care services through qualification of health care promoted by professionals and direct access of users, especially in low-income countries, whose health indicators are worrisome.

'Escola Paulista de Medicina, Universidade Federal de São Paulo, São Paulo, SP, Brasil.

¿Escola Paulista de Enfermagem, Universidade Federal de São Paulo, São Paulo, SP, Brasil.

Conflitos de interesse: nada a declarar. 


\section{Resumen}

Objetivo: Describir los impactos de las aplicaciones móviles directamente a favor de la salud en las comunidades menos favorecidas que viven en países de bajos recursos o en desarrollo e indirectamente a través de los profesionales de la salud que atienden en estas áreas,

Métodos: Revisión integradora de la literatura en las bases de datos PubMed, SciELO y LILACS de estudios originales basados en evidencias, publicados entre 2010 y 2019, con texto completo disponible en portugués e inglés, mediante la pregunta orientadora: ¿Cuál es el impacto de las aplicaciones móviles en el cuidado directo e indirecto de la salud en poblaciones de bajos recursos y en desarrollo?, a partir de los descriptores: tecnología biomédica, información en salud y aplicaciones móviles, este último presente en todos los cruces. La estrategia de búsqueda, selección y categorización de los estudios fue realizada mediante la lectura de un investigador.

Resultados: Las aplicaciones demostraron beneficios con relación al diagnóstico de enfermedades, adherencia de los usuarios al tratamiento, seguimiento de los pacientes por parte de los profesionales de la salud y autocuidado, lo que constituye una importante herramienta para el monitoreo y gestión de enfermedades.

Conclusión: La tecnología móvil tiene el potencial de mejorar los servicios de atención primaria mediante la cualificación de los cuidados de la salud promovidos por los profesionales y el acceso directo del usuario, sobre todo en países de bajos recursos, cuyos indicadores de salud son preocupantes.

\section{Introdução}

É indubitável o poder que a tecnologia exerce na sociedade contemporânea. Os avanços recentes na área da informática possibilitaram a transformação da internet num meio comum de informação, entretenimento e comunicação, permitindo a conexão de dados de diversas formas. Neste cenário de avanço tecnológico, destacam-se os dispositivos móveis, como smartphones e tablets, cujas popularidades permeiam a vida de usuários de todas as idades. ${ }^{(1)}$

$\mathrm{O}$ crescimento do uso de smartphones tem influenciado diretamente também a rotina dos brasileiros. Em 2014, o Android (89,1\%) era o sistema operacional mais usado, seguido por iOS (5,2\%) e Windows Phone (3,8\%), atingindo 107 milhóes de usuários, o que representou $53 \%$ da população nacional, colocando-o na quinta posição na lista dos países que mais acessam a rede, após China, Estados Unidos, Índia e Japão. ${ }^{(2)}$ Já em 2017, cerca de 67\% da população brasileira fazia uso da Internet, acesso com preferência via computador, quando comparado a smartphones ou $t a-$ blets; no entanto, a tendência é que nos próximos anos os smartphones ultrapassem os computadores. ${ }^{(3)}$

Os aplicativos, através dos dispositivos móveis não portáteis e não acessíveis, têm facilitado a vida das pessoas, ampliando o impacto da tecnologia na sociedade, permitindo a expansão da portabilidade e flexibilidade para vários tipos de relaçóes, como um meio de automatizaçáo de diversas tarefas, até então realizadas manualmente, despertando o interesse de muitos para o desenvolvimento de aplicativos capazes de solucionar problemas do cotidiano, aumentando o investimento por parte das empresas em soluçóes e ferramentas que facilitem o processo de desenvolvimento. ${ }^{(4)}$

Considerando a solução de problemas do cotidiano, açôes desenvolvidas em situaçôes sólidas da vida são requisitos universais de desenvolvimento e de alteraçôes da saúde. Neste contexto está o autocuidado, que corresponde à prática de atividade desenvolvida pela pessoa em benefício próprio fundamentada na manutenção da vida, do bem-estar e da saúde, ${ }^{(5)}$ pode ser estabelecido como a prática de atividades que beneficiam o aperfeiçoamento e amadurecem as pessoas que o inicia e o desenvolve dentro de espaços de tempo específicos, no intuito da promoção da vida e do bem-estar pessoal. ${ }^{(6)}$

Por esta razão, a redução da limitação da mobilidade constitui a principal característica dos dispositivos móveis, qualidade esta, fundamental para recursos empregados na assistência em saúde, considerando as peculiaridades inerentes ao trabalho desempenhado pelos profissionais dessa área ${ }^{(7)} \mathrm{e} \mathrm{a}$ autonomia pretendida aos pacientes no desenvolvimento do autocuidado.

Estas consideraçóes possibilitam uma reflexão sobre como os aplicativos móveis têm sido utilizados diretamente em favor da saúde das comunidades menos favorecidas, portanto, este estudo tem como objetivo descrever os impactos dos aplicativos móveis diretamente em favor da saúde das comunidades menos favorecidas que vivem em países de baixa renda ou em desenvolvimento e, indiretamente, por meio dos profissionais de saúde que prestam assistência nestas áreas. 
Tratou-se de uma revisão integrativa, por meio da identificação, análise e síntese dos resultados de estudos sobre o mesmo assunto. Este tipo de revisão permite a síntese e análise de pesquisas desenvolvidas e publicadas sobre determinado assunto, tanto pelo método quantitativo quanto qualitativo, possibilitando a compreensão mais abrangente de determinado fenômeno, capaz de subsidiar a tomada de decisão, baseada nas evidências científicas. ${ }^{(8)}$

As etapas desta revisão foram fundamentadas em um protocolo previamente estabelecido, visando manter o rigor científico e metodológico, a saber: 1) elaboração da pergunta de pesquisa; 2) definição dos critérios de inclusão de estudos e seleção da amostra; 3) representação dos estudos selecionados em formato de tabelas, considerando todas as características em comum (coleta de dados); 4) análise crítica dos estudos incluídos, identificando diferenças e conflitos; 5) interpretação/discussão dos resultados; 6) apresentação dos resultados com exposição das evidências encontradas.

Para responder à questão norteadora da revisão "Qual o impacto dos aplicativos móveis no cuidado direto e indireto em saúde em populaçóes de baixa renda e em desenvolvimento?", realizou-se a busca bibliográfica das publicaçôes indexadas nas bases de dados PubMed, SciELO e LILACS, a partir dos seguintes descritores: aplicativos móveis, tecnologia biomédica e informação em saúde. A estratégia de busca e seleção dos estudos foi realizada por um pesquisador.

Os critérios de inclusão dos estudos foram pesquisas originais publicadas entre 2010 e 2019, baseadas em evidências em língua portuguesa e inglesa, disponíveis na íntegra. Os critérios de exclusão foram duplicidade dos artigos, estudos de caso e estudos fora do tema abordado e recorte temporal. Após leitura do material os dados foram agrupados em categorias.

Os dados dos estudos selecionados para análise foram sintetizados mediante ano de publicação, autor, tipo de estudo, objeto de estudo, objetivo e consideraçóes do estudo, descritos em sessão dedicada.

\section{Resultados}

\section{Descrição das características do estudo}

Foram encontrados 557 artigos. Destes, 239 (43\%) se repetiam nas bases de dados e 300 (54\%) não correspondiam à temática, restando nove artigos, sendo um (10\%) da MEDLINE e oito (90\%) da PUBMED, como ilustrados na figura 1 .

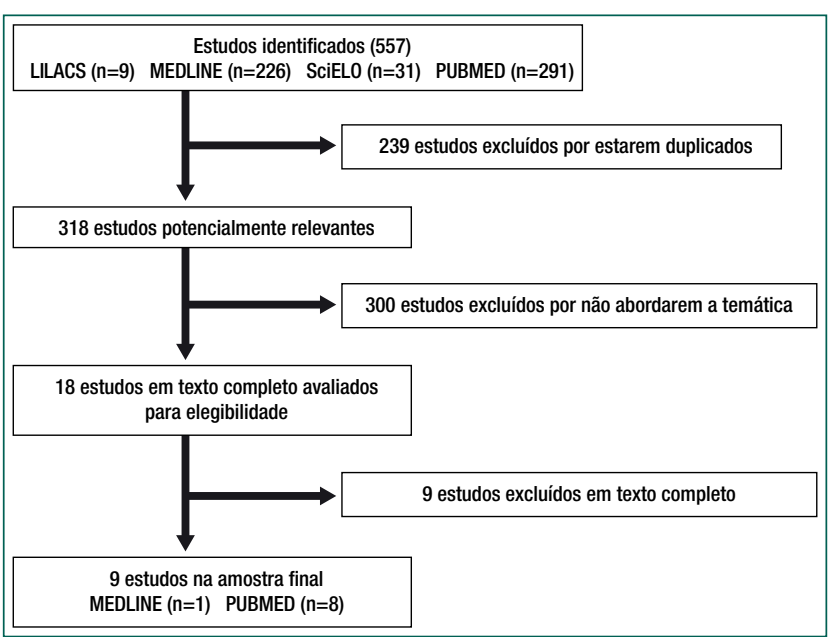

Figura 1. Fluxograma da seleção dos artigos incluídos na revisão integrativa

Os artigos foram categorizados por semelhança nos objetivos e temas de estudo: benefícios dos aplicativos à saúde dos usuários em decorrência da facilidade de acesso à informação em saúde $(n=5)$ e benefícios dos aplicativos que dão suporte aos profissionais de saúde que prestam assistência a populaçóes de baixa renda e em regióes em desenvolvimento, com precárias condiçôes $(n=4)$.

As publicaçóes selecionadas com a descrição dos impactos dos aplicativos móveis sobre a saúde nas sociedades menos favorecidas segundo ano de publicação, autoria, objetivo do estudo, método utilizado, população estudada, consideraçóes e influência dos aplicativos estão sintetizadas no quadro 1 .

De modo geral, pode-se inferir, pelos resultados apresentados nos estudos selecionados que o uso de aplicativos pode servir como recurso tecnológico trazendo impacto positivo direto para a saúde, sobretudo, em populaçóes de baixa renda ou com acesso dificultado à internet ou indireto por meio dos profissionais que prestam assistência à saúde nestas regióes. 
Quadro 1. Integração das publicações que retratam o uso de aplicativos móveis voltados para o cuidado em saúde nas sociedades menos favorecidas

\begin{tabular}{|c|c|c|c|c|c|c|c|}
\hline Ano & Autor & Estudo / referência & $\begin{array}{l}\text { Tipo de } \\
\text { estudo }\end{array}$ & $\begin{array}{l}\text { Objeto de } \\
\text { estudo }\end{array}$ & Objetivo & Considerações do estudo & $\begin{array}{l}\text { Influência dos } \\
\text { aplicativos }\end{array}$ \\
\hline 2019 & $\begin{array}{l}\text { Menezes } \\
\text { et al }{ }^{(9)}\end{array}$ & $\begin{array}{l}\text { Use of a Mobile Phone } \\
\text { App to Treat Depression } \\
\text { Comorbid With Hypertension } \\
\text { or Diabetes: A Pilot Study in } \\
\text { Brazil and Peru }\end{array}$ & $\begin{array}{l}\text { Estudo } \\
\text { experimental }\end{array}$ & $\begin{array}{l}\text { Pacientes } \\
\text { com } \\
\text { sinais de } \\
\text { depressão }\end{array}$ & $\begin{array}{l}\text { Explorar a eficácia potencial de uma } \\
\text { intervenção de saúde móvel para ajudar } \\
\text { pessoas com sintomas depressivos e } \\
\text { comorbidade hipertensão ou diabetes }\end{array}$ & $\begin{array}{l}0 \text { aplicativo para celular demonstrou- } \\
\text { se viável no auxílio do tratamento de } \\
\text { pacientes com sintomas depressivos } \\
\text { comórbidos nos países de baixa e } \\
\text { média renda. }\end{array}$ & $\begin{array}{l}\text { Importante redução } \\
\text { dos sintomas } \\
\text { depressivos de } \\
\text { pacientes diabéticos e } \\
\text { hipertensos. }\end{array}$ \\
\hline 2019 & $\begin{array}{l}\text { Rajbhandari } \\
\text { et al (10) }\end{array}$ & $\begin{array}{l}\text { Epilepsy field workers, a } \\
\text { smartphone application } \\
\text { and telephone } \\
\text { telemedicine: Safe and } \\
\text { effective epilepsy care in } \\
\text { rural Nepal. }\end{array}$ & $\begin{array}{l}\text { Estudo } \\
\text { experimental }\end{array}$ & $\begin{array}{l}\text { Pacientes } \\
\text { com sinais e } \\
\text { sintomas de } \\
\text { epilepsia }\end{array}$ & $\begin{array}{l}\text { Propor um novo modelo de cuidado, a partir } \\
\text { do desenvolvimento de um aplicativo para } \\
\text { smartphone para diagnóstico da epilepsia a } \\
\text { partir de crises }\end{array}$ & $\begin{array}{l}0 \text { aplicativo propiciou considerável auxílio } \\
\text { no diagnóstico da epilepsia, bem como } \\
\text { na interrupção e redução das crises } \\
\text { propiciadas pela epilepsia, recebendo a } \\
\text { preferência dos pacientes a esse serviço } \\
\text { ofertado, evitando deslocamentos aos } \\
\text { serviços médicos para o diagnóstico. }\end{array}$ & $\begin{array}{l}\text { Agilidade no } \\
\text { diagnóstico; } \\
\text { Expressiva redução } \\
\text { dos deslocamentos } \\
\text { dos pacientes para } \\
\text { acesso ao serviço } \\
\text { médico. }\end{array}$ \\
\hline 2017 & $\begin{array}{l}\text { Carroll } \\
\text { et al }{ }^{(11)}\end{array}$ & $\begin{array}{l}\text { Who Uses Mobile Phone } \\
\text { Health Apps and Does Use } \\
\text { Matter? A Secondary Data } \\
\text { Analytics Approach. }\end{array}$ & $\begin{array}{l}\text { Estudo quase } \\
\text { experimental, } \\
\text { transversal, } \\
\text { de associação } \\
\text { de dados }\end{array}$ & $\begin{array}{l}\text { Usuários de } \\
\text { aplicativos }\end{array}$ & $\begin{array}{l}\text { Descrever as características sociais e } \\
\text { demográficas associadas ao uso de } \\
\text { aplicativos de saúde em uma recente } \\
\text { amostra representativa dos EUA; avaliar } \\
\text { atitudes e comportamentos dos usuários } \\
\text { de aplicativos de saúde para promoção } \\
\text { da saúde; examinar a associação entre o } \\
\text { uso de aplicativos relacionados à saúde e } \\
\text { as diretrizes recomendadas para ingestão } \\
\text { de frutas e hortaliças e atividade física, } \\
\text { informando as populações mais e menos } \\
\text { propensas a usar aplicativos de saúde. }\end{array}$ & $\begin{array}{l}\text { Apesar da falta de evidências de eficácia } \\
\text { clínica e integração com o sistema de } \\
\text { saúde e da necessidade de avaliação } \\
\text { formal e revisão e potenciais ameaças à } \\
\text { segurança e privacidade, os aplicativos } \\
\text { podem melhorar a saúde dos usuários, } \\
\text { além de fornecerem informações sobre } \\
\text { as populações mais e menos propensas } \\
\text { ao uso de aplicativos de saúde para } \\
\text { orientar intervenções clínicas por meio } \\
\text { de comerciais e programas de saúde } \\
\text { pública. }\end{array}$ & $\begin{array}{l}\text { Valioso progresso na } \\
\text { saúde dos usuários de } \\
\text { aplicativos. }\end{array}$ \\
\hline 2017 & $\begin{array}{l}\text { Uddin } \\
\text { et al }{ }^{(12)}\end{array}$ & $\begin{array}{l}\text { Impact of mobile phone- } \\
\text { based technology to } \\
\text { improve health, population } \\
\text { and nutrition services in } \\
\text { Rural Bangladesh: a study } \\
\text { protocol }\end{array}$ & $\begin{array}{l}\text { Estudo } \\
\text { experimental }\end{array}$ & $\begin{array}{l}\text { Mulheres } \\
\text { de uma } \\
\text { comunidade } \\
\text { rural de } \\
\text { Bangladesh }\end{array}$ & $\begin{array}{l}\text { Desenvolver, testar e avaliar o impacto de } \\
\text { um mecanismo de estratégias de saúde } \\
\text { móvel sobre melhoria da saúde reprodutiva e } \\
\text { planeamento familiar, saúde materna, neonatal } \\
\text { e infantil, gestão integrada de doenças infantis, } \\
\text { programa de vacinação e outros serviços de } \\
\text { saúde primários de comunidades nas áreas } \\
\text { rurais de Bangladesh. }\end{array}$ & $\begin{array}{l}\text { A tecnologia baseada em telefone } \\
\text { celular tem o potencial de melhorar } \\
\text { os serviços de atenção primária à } \\
\text { saúde por agilizar aos profissionais o } \\
\text { acesso às informações e estratégias } \\
\text { de atendimento aos serviços de saúde } \\
\text { materno-infantil, em países de baixa } \\
\text { renda. }\end{array}$ & $\begin{array}{l}\text { Melhoria relevante da } \\
\text { cobertura dos serviços } \\
\text { de atenção primária } \\
\text { à saúde, num país } \\
\text { de extrema pobreza, } \\
\text { reduzindo a busca } \\
\text { pela à saúde de média } \\
\text { complexidade }\end{array}$ \\
\hline 2013 & $\begin{array}{l}\text { Blank } \\
\text { et al }{ }^{(13)}\end{array}$ & $\begin{array}{l}\text { Quality of prenatal and } \\
\text { maternal care: bridging the } \\
\text { know-do gap" (QUALMAT } \\
\text { study): an elecronic clinical } \\
\text { decision support system for } \\
\text { rural Sub-Saharan Africa) }\end{array}$ & $\begin{array}{l}\text { Estudo } \\
\text { experimental } \\
\text { controlado e } \\
\text { randomizado }\end{array}$ & $\begin{array}{l}\text { Profissionais } \\
\text { da área da } \\
\text { saúde nas } \\
\text { regiões } \\
\text { rurais }\end{array}$ & $\begin{array}{l}\text { Melhorar o desempenho e a motivação dos } \\
\text { trabalhadores de saúde rurais e a qualidade } \\
\text { dos serviços de cuidados desenvolvidos no } \\
\text { nível primário de atenção à saúde materna } \\
\text { no Gana, Burkina Faso, e Tanzânia. }\end{array}$ & $\begin{array}{l}0 \text { sistema informatizado de apoio } \\
\text { à decisão clínica foi introduzido } \\
\text { nos centros rurais onde atuam } \\
\text { profissionais de saúde de diferentes } \\
\text { níveis educacionais, em função do } \\
\text { cenário subdesenvolvido do país, } \\
\text { com expressiva melhora de seu } \\
\text { desempenho. }\end{array}$ & $\begin{array}{l}\text { Enriquecimento } \\
\text { da qualidade ddos } \\
\text { cuidados em saúde } \\
\text { na atenção primária } \\
\text { promovidos por } \\
\text { profissionais que } \\
\text { atuam em locais } \\
\text { subdesenvolvidos. }\end{array}$ \\
\hline 2013 & $\begin{array}{l}\text { Mitchell } \\
\text { et al }{ }^{(14)}\end{array}$ & $\begin{array}{l}\text { Using electronic technology } \\
\text { to improve clinical } \\
\text { care - results from a } \\
\text { before-after cluster trial } \\
\text { to evaluate assessment } \\
\text { and classification of sick } \\
\text { children according to } \\
\text { Integrated Management } \\
\text { of Childhood IIIness (IMCI) } \\
\text { protocol in Tanzania) }\end{array}$ & $\begin{array}{l}\text { Estudo } \\
\text { experimental }\end{array}$ & $\begin{array}{l}\text { Profissionais } \\
\text { da saúde }\end{array}$ & $\begin{array}{l}\text { Estimar o impacto da tecnologia eletrônica } \\
\text { na adesão aos protocolos da Atenção } \\
\text { Integrada às Doenças Prevalentes na } \\
\text { Infância, comparados aos atuais protocolos } \\
\text { baseados em papel na Tanzânia }\end{array}$ & $\begin{array}{l}0 \text { estudo demonstrou o benefício da } \\
\text { tecnologia em fornecer um padrão } \\
\text { comprovado de cuidados em uma } \\
\text { área rural e semi-urbana da Tanzânia, } \\
\text { país de pobreza extrema, a partir } \\
\text { da qualidade de atendimento dos } \\
\text { profissionais que o utilizaram. }\end{array}$ & $\begin{array}{l}\text { Qualificação do } \\
\text { atendimento clínico } \\
\text { de profissionais que } \\
\text { atuam em países de } \\
\text { baixa renda. }\end{array}$ \\
\hline 2012 & $\begin{array}{l}\text { Mitchell } \\
\text { et al(15) }\end{array}$ & $\begin{array}{l}\text { Perceived improvement } \\
\text { integrated management } \\
\text { of childhood illness } \\
\text { implementation through } \\
\text { use of mobile technology: } \\
\text { qualitative evidence from a } \\
\text { pilot study in Tanzania) }\end{array}$ & $\begin{array}{l}\text { Relato de } \\
\text { experiência }\end{array}$ & $\begin{array}{l}\text { Provedores } \\
\text { de cuidados } \\
\text { de saúde e } \\
\text { cuidadores } \\
\text { da Gestão } \\
\text { Integrada } \\
\text { Eletrônica } \\
\text { de Doenças } \\
\text { Infantis } \\
\end{array}$ & $\begin{array}{l}\text { Examinar a percepção de cuidadores e } \\
\text { profissionais de saúde sobre o tratamento } \\
\text { de doenças infantis quando comparado com } \\
\text { as formas de papel convencionais por meio } \\
\text { de em entrevistas qualitativas. }\end{array}$ & $\begin{array}{l}\text { Percepção dos provedores de cuidados } \\
\text { em saúde de receberem mais } \\
\text { informações fizeram a utilização dos } \\
\text { assistentes pessoais digitais, ampliando } \\
\text { o seu conhecimento sobre o tratamento } \\
\text { das doenças infantis em um país de } \\
\text { extrema pobreza. }\end{array}$ & $\begin{array}{l}\text { Agilidade no acesso } \\
\text { à informação sobre } \\
\text { o tratamento a ser } \\
\text { realizado, ofertado } \\
\text { com mais agilidade, } \\
\text { sobretudo em locais de } \\
\text { baixa renda. }\end{array}$ \\
\hline 2012 & $\begin{array}{l}\text { Pellegrini } \\
\text { et al }{ }^{(16)}\end{array}$ & $\begin{array}{l}\text { A smartphone supported } \\
\text { weight loss program: } \\
\text { design of the ENGAGED } \\
\text { randomized controlled trial }\end{array}$ & $\begin{array}{l}\text { Estudo } \\
\text { experimental }\end{array}$ & $\begin{array}{l}\text { Pessoas } \\
\text { portadoras } \\
\text { de } \\
\text { obesidade }\end{array}$ & $\begin{array}{l}\text { Examinar a viabilidade e a eficácia de um } \\
\text { programa de perda de peso apoiado por } \\
\text { smartphones. }\end{array}$ & $\begin{array}{l}\text { Houve significativa redução da } \\
\text { obesidade nos pacientes que utilizaram } \\
\text { a tecnologia. }\end{array}$ & $\begin{array}{l}\text { Mudança de hábitos e } \\
\text { comportamentos dos } \\
\text { usuários por meio das } \\
\text { metas do pelo aplicativo. }\end{array}$ \\
\hline 2011 & $\begin{array}{l}\text { Florez- } \\
\text { Arango } \\
\text { et al(17) }\end{array}$ & $\begin{array}{l}\text { Performance factors of } \\
\text { mobile rich media job } \\
\text { aids for community health } \\
\text { workers }\end{array}$ & \begin{tabular}{|l|} 
Estudo \\
experimental \\
controlado e \\
randomizado
\end{tabular} & $\begin{array}{l}\text { Agentes } \\
\text { comunitários } \\
\text { de saúde }\end{array}$ & $\begin{array}{l}\text { Estudar e analisar os possíveis benefícios } \\
\text { no desempenho de agentes comunitários } \\
\text { de saúde usando diretrizes clínicas prontas } \\
\text { para o atendimento implementadas como } \\
\text { auxílios interativos de trabalho rich media } \\
\text { em plataformas móveis de pequeno formato. }\end{array}$ & $\begin{array}{l}\text { Aceitação generalizada deste recurso } \\
\text { pelos agentes comunitários de saúde } \\
\text { com níveis educacionais mais baixos } \\
\text { e que atendem a populações com } \\
\text { alfabetização muito pobre indicando } \\
\text { perspectivas encorajadoras para as } \\
\text { tecnologias de saúde móvel em geral e } \\
\text { o uso de diretrizes clínicas em telefones } \\
\text { móveis em particular }\end{array}$ & $\begin{array}{l}\text { Melhoria da assistência } \\
\text { em saúde em função } \\
\text { da facilidade de } \\
\text { acesso à informação } \\
\text { de profissionais que } \\
\text { atendem população } \\
\text { com baixo nível de } \\
\text { instrução em locais } \\
\text { subdesenvolvidos. }\end{array}$ \\
\hline
\end{tabular}




\section{Discussão}

Os aplicativos presentes nos dispositivos móveis, específicos para a áreas de saúde, também chamados de mobile health applications ou mHealth, atribuem significativa relevância como ferramentas tanto para pesquisadores quanto para profissionais de saúde quando considerados monitoramento e gerenciamento de doenças crônicas, que utilizam a comunicação wireless na prática clínica e suporte à saúde. ${ }^{(18)}$

Estes dispositivos apresentam benefícios sobre computadores tradicionais, sobretudo nos países em desenvolvimento, em função do seu menor custo de aquisição e à facilidade de uso inclusive para usuários com pouca experiência. ${ }^{(19)}$

Considerando auxílio no trabalho diagnóstico, um estudo desenvolvido com moradores de um distrito rural do Nepal, país de baixa renda onde 50\% dos casos de epilepsia não recebem tratamento e há poucos médicos, teve como objetivo o treinamento de moradores desta área como "trabalhadores de campo de epilepsia", fornecendo a eles a conscientização da epilepsia e suas consequências em suas comunidades, orientando-os a utilizarem um aplicativo de smartphone com capacidade de determinar a probabilidade de um episódio ser epiléptico, contatando um especialista em epilepsia por telefone. ${ }^{(10)}$

Outro estudo, desenvolvido com mulheres de baixa renda residentes em comunidades das regióes Centro-Oeste e costas leste e oeste dos Estados Unidos da América (EUA), objetivou compreender a extensão da adoção e uso de ferramentas digitais de saúde, além de identificar os principais fatores psicológicos percebidos no uso da tecnologia, obtendo como resultado o acesso da maioria em busca de informação em saúde: evidências indicam que indivíduos que experimentam dificuldades em acessar cuidados de saúde por razóes não relacionadas ao seu status social são mais propensos a relatar usar a internet para informaçóes de saúde, ratificando que os benefícios do uso de ferramentas digitais proporcionam benefícios aos indivíduos com recursos e habilidades para usar a tecnologia de forma eficaz. ${ }^{(20)}$

Tanto os celulares quanto os tablets possuem maior ingresso de mercado em países em desen- volvimento, quando a comparação é realizada com computadores. A redução de preço dos dispositivos móveis e dos planos de telefonia com acesso à internet produziu um aumento considerável no número de usuários conectados à rede móvel. No fim de 2014, 56,2\% dos celulares eram acessíveis à banda larga móvel no país, perfazendo mais de 148 milhôes de dispositivos conectados. Tais dados justificam os esforços na migração de serviços web para plataformas Android e iOS como experiência de ampliação e diversificação do público atingido, sendo que equipamentos baseados no sistema Android possuem custo mais baixo. ${ }^{(21)}$

Proporcionando, por meio da tecnologia, o rastreamento da gravidez e lembretes para as visitas e para o parto, um estudo desenvolvido com a população de Bangladesh aplicou o uso da tecnologia baseada em telefonia móvel à saúde com o intuito de aumentar o acesso das pessoas ao cuidado. Durante o parto, a tecnologia foi utilizada para consultas remotas no local de atendimento, facilitando o não só o encaminhamento, como também o acesso aos serviços de saúde, promovendo contato ágil e oportuno com os agentes comunitários de saúde (ACS) das suas respectivas áreas de trabalho, ${ }^{(12)}$ otimizando tempo e aproximando a parturiente dos cuidados necessários àquele momento.

No contexto brasileiro, o acesso universal aos serviços de saúde, bandeira de luta dos movimentos sociais garantido pela Constituição de 1988, constituiu-se em um dos direitos fundamentais de cidadania. Este acesso não retrata a simples utilização do serviço de saúde e sim, a oportunidade de dispor dos serviços em circunstâncias que permitam o uso apropriado dos mesmos em tempo hábil para o alcance de bons resultados de saúde a partir da equidade e da integralidade, ${ }^{(21)}$ no entanto, ainda constitui um grande desafio nas sociedades menos favorecidas.

No Quênia, país africano, as práticas de gestão de casos de malária dos trabalhadores de saúde frequentemente diferem das diretrizes nacionais. Diante deste cenário, o envio de lembretes de mensagens de texto enviados aos telefones móveis dos profissionais de saúde para a melhoria e a manutenção da adesão às diretrizes de tratamento para malária pediátrica 
ambulatorial foi testado, evidenciando que em ambientes com recursos limitados, o uso de mensagens de texto pode melhorar as práticas de gerenciamento de casos dos trabalhadores de saúde. ${ }^{(22)}$

$\mathrm{Na}$ Colômbia, um estudo desenvolvido pela Universidade de Antioquia, a partir da utilização das diretrizes clínicas apresentadas por meio de texto, áudio, imagens e vídeo em telefones celulares para ACS indicou perspectivas encorajadoras para as tecnologias de saúde móvel em geral e o uso de diretrizes clínicas em telefones móveis em particular, para apoiar a saúde global, quando considerada a assistência em saúde, ${ }^{(17)}$ em função da facilidade do acesso à informação dos profissionais com baixo nível educacional que dão assistência à população em precárias condiçóes de instrução, comprometendo a interpretação das informaçóes recebidas para o cuidado em saúde.

Diferentes gargalos marcam a atenção especializada, quando considerado o acesso à saúde. A dificuldade para a garantia de acesso a serviços especializados depende do modelo de atenção em vigor, da resolutividade da atenção primária, melhoria da resolutividade da atenção primária, sobretudo onde não há profissionais de saúde suficientes, além do dimensionamento e organização da oferta dos serviços. ${ }^{(23)}$

Na Tanzânia, um estudo, cujo objetivo foi estimar o impacto da tecnologia eletrônica na adesão aos protocolos da Atenção Integrada às Doenças Prevalentes na Infância, comparados aos atuais protocolos baseados em papel demonstrou o seu benefício em fornecer um padrão comprovado de cuidados em uma área rural e semi-urbana, qualificando o atendimento clínico em países de baixa renda. ${ }^{(14)}$

A fim de controlar o peso de pessoas adultas por meio de um programa chamado SmarDiet desenvolvido por pesquisadores, a tecnologia proporcionou regularidade na alimentação dos usuários, bem como aumento na prática de exercício físico e diminuição na composição corporal, que afirmaram que tal tipo de ferramenta é de fácil utilização e acesso, bem como a forma interessante e motivadora de seu conteúdo. ${ }^{(24)}$

Já, um outro programa desenvolvido para perda de peso através de uma estratégia denominada E-Networks Guiding Adherence to Goals in Exercise and Diet - ENGAGED, estabeleceu metas para a automonitorização dos seus objetivos e modificação do comportamento utilizando-se de smartphones. O programa demonstrou que esta intervenção inovadora de perda de peso integra a teoria às tecnologias móveis emergentes, reafirmando sua eficácia e viabilidade como suportes em programas para perda de peso, possibilitando a redução de custos de programas e manutenção dos resultados eficazes. ${ }^{(16)}$

O QUALMAT, projeto de investigação financiado pela União Europeia que avalia a qualidade dos cuidados pré-natais e maternais, promoveu um estudo cuja proposta foi melhorar o desempenho e a motivação dos trabalhadores de saúde rurais, além da melhoria da qualidade dos serviços de cuidados desenvolvidos no nível primário de atenção à saúde materna no Gana, Burkina Faso e Tanzânia, países africanos que mantêm sua qualidade de atendimento deficiente constatada por meio das altas taxas de mortalidade materna, apesar dos fortes esforços para melhoria dos cuidados. Por meio de um programa capaz de ser executado em qualquer equipamento padrão desenvolvido com base na avaliação da situação dos cuidados de saúde, o estudo afirmou que um sistema informatizado de apoio à decisão clínica é essencial para centros rurais onde atuam profissionais de saúde de diferentes níveis educacionais. ${ }^{(13)}$

Outro estudo qualitativo realizado na Tanzânia, cujo objetivo foi examinar a percepção do provedor de cuidados de saúde e cuidador da Gestão Integrada Eletrônica de Doenças Infantis quando comparada às formas de papel convencionais no diagnóstico e tratamento de doenças infantis observou uma melhoria no serviço prestado pelos provedores que utilizavam os assistentes pessoais digitais, por serem mais bem informados. ${ }^{(15)}$

É notório que todos os âmbitos sociais no mundo são beneficiados com a realidade da tecnologia mediando a comunicação e a informação. A internet tem estimulado profundas mutaçóes nas dinâmicas sociais, culturais e econômicas, superando a expectativa de ser um meio de comunicação, uma tecnologia ou mesmo um meio de interação social somente. ${ }^{(25)}$

A facilidade que os aplicativos tem proporcionado à vida de muitas pessoas permite uma ampla extensão de impacto no mundo da tecnologia, por meio da portabilidade e flexibilidade para vários tipos de negócios, 
sejam uma automação residencial, saúde e bem-estar, armazenamento de informaçóes na nuvem, meios de expor o conhecimento dentre outros, ${ }^{(18)}$ no entanto, a necessidade da participação dos profissionais da saúde tanto no desenvolvimento quanto na supervisão desses equipamentos tecnológicos são essenciais para a garantia da qualidade necessária quando considerada a saúde, em seu amplo conceito.

Buscando fornecer informaçôes atualizadas sobre as populaçóes mais e menos propensas a usar aplicativos de saúde para orientar intervençóes clínicas com objetivo principal, um estudo transversal revelou que os aplicativos de saúde apresentam limitaçôes, como falta de evidências de eficácia clínica, integração com o sistema de saúde, necessidade de avaliação formal e revisão, além da vulnerabilidade de potenciais ameaças à segurança e privacidade, náo obstante a desmedida adesáo à propriedade de telefones celulares, ao uso de aplicativos de saúde e seu potencial para melhoria da saúde. ${ }^{(11)}$

Em países subdesenvolvidos, de baixa e média renda, com investimentos insuficientes destinados à sáude é comum o subdiagnósico da depressão na atenção primária à saúde, potencializado quando o paciente apresenta distúrbios físicos crônicos, comprometendo também o tratamento devido à escassez de recursos financeiros, materiais e humanos. Intervenções suportadas pelas tecnologias de saúde móvel podem mitigar os desdobramentos indesejáveis, por meio da oferta de alternativas potencialmente viáveis e acessíveis para o tratamento da depressão entre indivíduos com distúrbios crônicos. ${ }^{(9)}$

Diante das evidências aqui discutidas, a repercussão dos aplicativos para a saúde, desenvolvidos de maneira adequada pode ser positiva, contribuindo em diferentes delineamentos para a melhoria da vida das pessoas, especialmente em locais de baixa renda, em regióes isoladas dos grandes centros e países subdesenvolvidos e em desenvolvimento, no entanto, seu potencial ainda precisa ser melhor explorado, tanto em comunidades completamente conectadas como no caso dos grandes centros urbanos, quanto em outras isoladas seja pelo aspecto geográfico, como no caso de cidades do interior ou mesmo por questóes culturais, como comunidades indígenas e quilombolas.

\section{Conclusão}

Os resultados encontrados nesta revisão permitem considerar que a tecnologia móvel tem o potencial de melhorar os serviços de atenção primária à saúde por meio da qualificação dos cuidados em saúde promovidos pelos profissionais e também pelo acesso direto do usuário à tecnologia, sobretudo em países de baixa renda, cujos indicadores de saúde são preocupantes. Intervenções baseadas em telefonia móvel para melhorar a qualidade e cobertura dos serviços devem ser monitoradas, principalmente, por aqueles que utilizam o dispositivo para alcance de metas a longo prazo, sendo supervisionados periodicamente por profissionais de saúde capacitados, objetivando verificar a sustentabilidade das mudanças na busca pela saúde, ou seja, das açôes efetivadas voltadas para o autocuidado, considerando o contexto do usuário. A necessidade do usuário, bem como a sua capacidade de lidar com as tecnologias devem ser acompanhadas e validadas por profissionais capacitados, de modo que o desenvolvimento e a aplicação dessas mídias sejam sustentados por pesquisas científicas e metodologias rigorosas, sobretudo, por representar um amplo campo de investigação pautado e estruturado no conhecimento, na ética e no respeito, ratificando a equidade proporcionada por ele, quando consideradas as condiçốes dos sujeitos à ele destinado ou que recebem o cuidado por ele proporcionado.

\section{Referências}

1. Duda R, Silva SC. Desenvolvimento de aplicativos para Android com uso do App inventor: uso de novas tecnologias no processo de ensino aprendizagem em matemática. Rev Conexão. 2015;11(3):310-23.

2. Cappellozza A, Moraes GH, Muniz LM. Uso pessoal das tecnologias no trabalho: motivadores e efeitos à distração profissional. Rev Adm Contemp. 2017;21(5):605-26.

3. Formagini TDB, Ervilha RR, Machado NM, Andrade BABB, Gomide HP, Ronzani TM. Revisão dos aplicativos de smartphones para cessação de tabagismo disponíveis em língua portuguesa. Cad Saúde Pública. 2017; 33(2). Doi: https://doi.org/10.1590/0102$311 \times 00178215$

4. Silva AM, Paiva IG, Fortes DX. Desenvolvimento de aplicativo para Android com uso do MIT APP inventor. Rev Cient Fasete. 2017;11(13):191-203. 
5. Trettene AD, Fontes CM, Razera AP, Gomide MR. Impact of promoting self-care in nursing workload. Rev Esc Enferm USP. 2016;50(4):635-41.

6. Queirós PJ, Vidinha TS, Almeida Filho AJ. Self-care: orem's theoretical contribution to the Nursing discipline and profession. Rev Enferm Ref. 2014;4(3):157-64.

7. Vêscovi SJB, Primo CC, Sant' Anna HC, Bringuete MEO, Rohr RV, Prado TN, et al. Aplicativo móvel para a avaliação dos pés de pessoas com diabete mellitus. Acta Paul Enferm. 2017; 30(6):607-13. D0I: http:// dx.doi.org/10.1590/1982-0194201700087

8. Whittemore R, Knafl K. The integrative review: updated methodology. J Adv Nurs. 2005;52(5):546-53.

9. Menezes P, Quayle J, Garcia CH, Silva S, Brandt LR, Diez-Canseco F, et al. Use of a mobile phone app to treat depression comorbid with hypertension or diabetes: a pilot study in Brazil and Peru. JMIR Ment Health. 2019;6(4):e11698.

10. Rajbhandari H, Joshi S, Malakar S, Paudel P, Jain P, Uppadaya K, et al. Epilepsy field workers, a smartphone application and telephone telemedicine: safe and effective epilepsy care in rural Nepal. Seizure. 2019;64:54-8.

11. Carroll JK, Moorhead A, Bond R, LeBlanc WG, Petrella RJ, Fiscella K. Who uses mobile phone health apps and does use matter? A secondary data analytics approach. J Med Internet Res. 2017;19(4):e125.

12. Uddin J, Biswas T, Adhikary G, Ali W, Alam N, Palit R, et al. Impact of mobile phone-based technology to improve health, population and nutrition services in Rural Bangladesh: a study protocol. BMC Med Inform Decis Mak. 2017;17(1):101.

13. Blank A, Prytherch H, Kaltschmidt J, Krings A, Sukums F, Mensah N, et al. "Quality of prenatal and maternal care: bridging the know-do gap" (QUALMAT study): an electronic clinical decision support system for rural Sub-Saharan Africa. BMC Med Inform Decis Mak. 2013;13(1):44.

14. Mitchell M, Hedt-Gauthier B, Msellemu D, Nkaka M, Lesh N. Using electronic technology to improve clinical care - results from a beforeafter cluster trial to evaluate assessment and classification of sick children according to Integrated Management of Childhood Illness (IMCI) protocol in Tanzania. BMC Med Inform Decis Mak. 2013;13:95.

15. Mitchell M, Getchell M, Nkaka M, Msellemu D, Van Esch J, HedtGauthier B. Perceived improvement in integrated management of childhood illness implementation through use of mobile technology: qualitative evidence from a pilot study in Tanzania. J Health Commun. 2012; 17:118-27.
16. Pellegrini CA, Duncan JM, Moller AC, Buscemi J, Sularz A, DeMott $A$, et al. A smartphone-supported weight loss program: design of the ENGAGED randomized controlled trial. BMC Public Health. 2012;12(1):1041-51.

17. Florez-Arango JF, lyengar MS, Dunn K, Zhang J. Performance factors of mobile rich media job aids for community health workers. J Am Med Inform Assoc. 2011;18(2):131-7.

18. Lamboglia CM, Silva CA, Vasconcelos Filho JE, Carvalho LM, Silva Júnior FVI, Mumford L. O vilão se torna mocinho: uma perspectiva inovadora da utilização das tecnologias de entretenimento e comunicação para a promoção e práticas em saúde. In: Santos ZM, Frota MA, Martins AB. Tecnologias em saúde: da abordagem teórica a construção e aplicação no cenário do cuidado. Fortaleza: EdUECE: 2016. p.43-63.

19. Romani LA, Magalhães GB, Evangelista SR. Desenvolvimento de aplicativos móveis em agricultura: Agritempo mobile. In: Congresso Brasileiro de Agroinformática, 10, 2015, Ponta Grossa. Uso de VANTs e sensores para avanços no agronegócio: Anais. Ponta Grossa: Universidade Estadual de Ponta Grossa; 2015.

20. Guendelman S, Broderick A, Mlo H, Gemmill A, Lindeman D. Listening to communities: mixed-method study of the engagement of disadvantaged mothers and pregnant women with digital health technologies. J Med Internet Res. 2017;19(7):e240.

21. Leite RA, Brito ES, Silva LM, Palha PF, Ventura CA. Access to healthcare information and comprehensive care: perceptions of users of a public service. Interface (Botucatu). 2014;18(51):661-71.

22. Zurovac D, Sudoi RK, Akhwale WS, Ndiritu M, Hamer DH, Rowe AK, et al. The effect of mobile phone text-message reminders on Kenyan health workers' adherence to malaria treatment guidelines: a cluster randomised trial. Lancet. 2011;378(9793):795-803.

23. Barreto ML, Teixeira MG, Bastos FI, Ximenes RA, Barata RB, Rodrigues LC. Successes and failures in the control of infectious diseases in Brazil: social and environmental context, policies, interventions, and research needs. Lancet. 2011;377(9780):1877-89.

24. Gopalan A, Makelarski JA, Garibay LB, Escamilla V, Merchant RM, Wolfe MB Sr, et al. Health-specific information and communication technology use and its relationship to obesity in high-poverty, urban communities: analysis of a population-based biosocial survey. J Med Internet Res. 2016;18(6):e182.

25. Rebelo $\mathrm{C}$. The use of the Internet and Facebook by the elders in Portugal: na exploraty study. Observ J. 2015;9(3):129-53. 